\title{
INDEX FOR THE EVALUATION OF SEGMENTATION (IAVAS): AN APPLICATION TO AGRICULTURE
}

\author{
J. C. Oliveira, A. R. Formaggio, J. C. N. Epiphanio, \\ and A. J. B. Luiz \\ Instituto Nacional de Pesquisas Espaciais (INPE), \\ Caixa Postal 515, CEP 12245-970, \\ São José dos Campos, SP, Brazil
}

\begin{abstract}
The use of segmentation algorithms for determining the boundaries of cultivated plots is an essential step in the process of agricultural land use classification using digital remote sensing imagery. Thus, the main objective of the present research was the development of a quantitative method (IAVAS) for evaluating the results of segmentation of space imagery. The proposed methodology defines criteria for selecting thresholds (area and similarity) for the segmentation algorithm (region-growing approach). From the results obtained it was verified that the quantitative methodology proposed provides a suitable and efficient way to identify the best segmentation thresholds for agricultural land use classification.
\end{abstract}

\section{INTRODUCTION}

Evaluation of the performance of segmentation algorithms is of fundamental importance because it provides objective and quantitative guidelines for selecting the best result and assists in the development of new algorithms (Zhang, 1996, 1997). In order to obtain a good classification based on regions, an effective segmentation of the subjects of interest is essential. However, Zhang (1996) has noted that, although several segmentation techniques are presented in the literature, few evaluation methods have been developed to judge the quality of the segmentation and the performance of the segmentation algorithms.

According to Woodcock and Harward (1992), in remote sensing the objective of segmentation is to define, in the studied image, regions that correspond to the objects present on the land surface. In this context, Woodcock et al. (1994), Zhang (1997), Borsotti et al. (1998) and Xu et al. (1998) have stated that the segmentation process consists of subdividing an image into homogeneous regions, according to some of its intrinsic characteristics that best represent the objects in the studied scene (such as, for example, the grey level of pixels or image texture).

According to Haralick and Shapiro (1984), the edges of segmented regions must be simple, non-shriveled, and have a distribution that is spatially coherent with the surface being studied. It is essential that the number of minor segments in the interior of these regions is small, and that the adjoining segments have values that are significantly different to be distinct in terms of the characteristic for which they are considered to be uniform.

Gonzalez and Wintz (1987) observed that, in general, the criteria for segmentation are established according to grey levels in the image, based on two intrinsic 
characteristics: discontinuity in the change of grey (sudden major changes in grey tone) and similarity within an aggregation of pixels due to the likeness of the neighbors' level of grey. Data involving area, average, variance, perimeter, and edge eccentricity and linearity are some of the variables analyzed by a generic segmentation algorithm.

INPE (2002) proposed the use of image segmentation prior to the classification phase, once edge information defines adjoining regions whose spectral and spatial properties are used to group pixels into homogeneous areas. In this way it is possible to overcome the limitations of point-based analysis that relies exclusively on the spectral characteristics of individual pixels during the classification process.

Zucker (1976) showed that these characteristics describe important aspects of the segmentation algorithms, but do not result in a unique algorithm for performing image fragmentation. Many segmentation algorithms have incorporated those conditions. Subsequently, Schoenmakers et al. (1991) suggested some changes, in order to modify algorithms with heuristics appropriate for each application, based on the needs of the end user.

The region-growing approach (RGA) for segmentation is an interactive process in which regions are built from individual pixels until all pixels are processed (Nascimento, 1997). The foliowing steps, according to Bins et al. (1996) describe the procedures of segmentation based on the region-growing approach: (1) the entire image is segmented into standard cells (one or more pixels); (2) each standard cell is compared, using a similarity scale, to its neighboring cells to determine whether they are similar; if similar, the cells are merged to form a fragment and the properties of this fragment are updated in preparation for the next comparison; (3) the fragments continue to grow and the algorithm continues to check all the fragment's neighbors until the fragment attains closure (is fully bounded); once this occurs the fragments become complete regions; (4) the next unassigned cell is analyzed and the preceding steps are repeated until all cells are tagged.

One disadvantage of this traditional procedure is that the interactions depend on the order of merging; that is, the growth of the second region will start only when the growth of the first region is complete. This requirement could produce different results depending on the initial region chosen in the process of segmentation.

The algorithm in the SPRING software (INPE, 2002) and used in the present research is based on the traditional region-growing approach (RGA), with some alterations to reduce the problem of dependence on the merging order (Bins et al., 1996). For each repetition a set of sub-images is defined and the most similar pairs of spatially adjoining regions are merged in each sub-image.

Bins et al. (1996) have defined the algorithm as follows: (1) at the beginning of the segmentation process a list of regions $\left\{\mathrm{R}_{\mathrm{i}}, \mathrm{i}=1,2, \ldots, n\right\}$ is created ( $n$ being the number of pixels in the image). Initially each region consists of a single unique pixel named the "seed"; for each $\mathrm{R}_{\mathrm{i}}$ region, its average vector and those of the neighboring regions are stored; (2) for each $\mathrm{R}_{\mathrm{i}}$ region the neighboring regions $N\left(\mathrm{R}_{\mathrm{i}}\right)$ are checked and the most similar neighboring region $\mathrm{R}_{\mathrm{k}} \in N\left(\mathrm{R}_{\mathrm{i}}\right)$ is chosen [if $\mathrm{D}\left(\mathrm{R}_{\mathrm{i}}, \mathrm{R}_{\mathrm{k}}\right)<\mathrm{T}(\mathrm{t})$, then $R_{k}$ is named $R_{i}$ 's "best neighbor" and the two regions are merged]; (3) the region is constantly merged with others, until all possible regions have been checked; $(4)$ the mean value of a region is updated each time it is merged with another; (5) the same 
procedure is repeated until no region lacks closure; (6) in a final step, small regions are merged into larger adjoining regions, according to an area threshold value defined by the user. According to the description above, $\mathfrak{R}$ represents a set of regions in the image and $R_{i} \in \Re$ is an element of this set.

$T(t)$ describes the threshold value below which two regions are considered similar in moment $t$, and $M_{i}$ is the vector of the average value in region $R_{i} \cdot D\left(R_{i}, R_{k}\right)=$ $\|\mathrm{Mi}-\mathbf{M k}\|$ is the Euclidean distance between the spectral average values in regions $\mathrm{R}_{\mathrm{i}}$ and $\mathbf{R}_{k}$, and $N(R)$ is the set of $\mathbf{R}$ neighboring regions ( $R$ itself not included). $\mathbf{R}_{k}$ is the neighboring region most similar to $\mathbf{R}_{\mathrm{i}}$ if $\mathrm{D}\left(\mathbf{R}_{\mathrm{i}}, \mathrm{R}_{\mathrm{k}}\right) \leq \mathrm{D}\left(\mathrm{R}_{\mathrm{i}}, \mathbf{R}_{\mathrm{l}}\right)$ for every $\mathrm{R}_{\mathrm{l}} \in N\left(\mathrm{R}_{\mathrm{i}}\right)$.

When the SPRING software is run, $T(t)=\alpha^{t} T(0)$ in this algorithm, with $T(0)>0$, $\mathrm{t}=0,1,2 \ldots$, and $\alpha<1$. This specification dictates that only very similar regions are initially merged.

The steps of the segmentation process using the region-growing algorithm implemented in the SPRING software are as follows:

1. Selection of the spectral bands to be used in the segmentation process. The number of bands is defined by the user, once the algorithm segments one or more bands at the same time. A condition for the utilization of more than one band is that all of them have the same geometric resolution.

2. Selection of the similarity and area thresholds that will define the expected result of the segmentation. The similarity threshold specifies that two regions are similar when the minimum value of the Euclidean distance between the average of the regions is lower than the established threshold. The area threshold represents the minimum area, in pixels, for a region to be individualized. Regions that are smaller than the established threshold are absorbed by adjoining regions that have the nearest spectral average. 1

In order to obtain a more precise segmentation of all the objects present in the studied scene, a quantitative and objective method for the evaluation of the results produced by the process of land surface digital image segmentation process has been developed. The assumed hypothesis is that, to determine the best result of the segmentation process, there is an optimal set of defining variables (similarity and area thresholds) of the segmentation algorithm, according to the region and kind of interest.

\section{METHODOLOGY}

A new way to evaluate quantitatively the segmentation algorithms, based on an empirical discrepancy method, is presented in this paper. The use of an objective evaluation index, here named Index for the Evaluation of Segmentation (IAVAS) is proposed. IAVAS consists of parameters that quantify the discrepancies between a given reference image and a set of segmented images obtained by the use of distinct pairs of similarity and area thresholds.

Basically, the method consists of optimizing the process of choosing the best pairs of similarity and area thresholds within the available space of options. These thresholds are necessary for the definition of the segmentation level that is responsible for the number and size of regions to be extracted, when the RGA algorithm is 


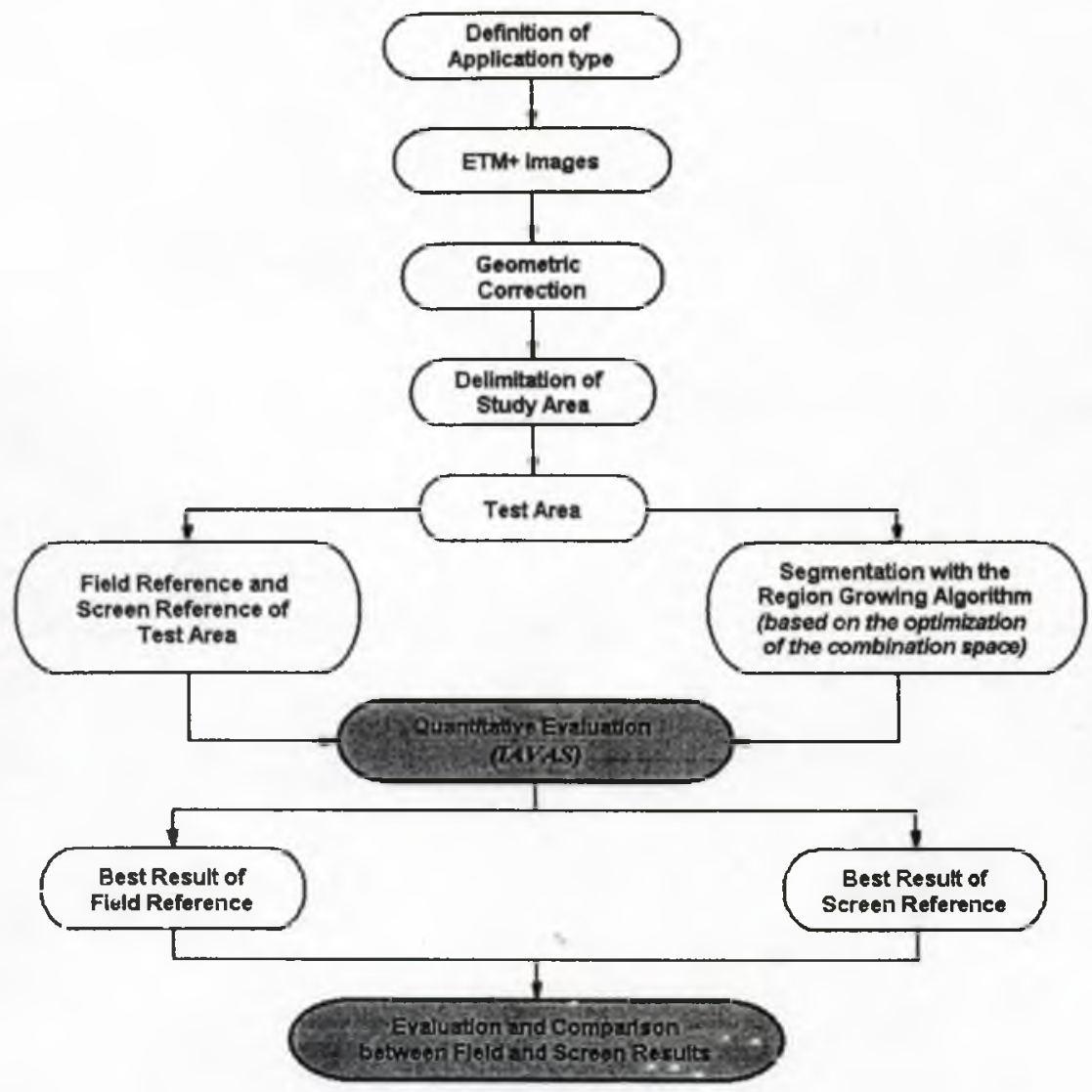

Fig. 1. Schematic diagram of methodology.

used. The steps followed in this methodology are depicted in Figure 1 and are detailed in subsequent sections of the paper.

\section{Defining the User's Application Type}

A fundamental step for the evaluation of the results obtained by the segmentation is the identification of the type of application that interests the user, which determines the reason for segmentation of the image. Zhang (1996) has noted that the type of application establishes the set of images to be used, the type of segmentation algorithm to be used, the objects to be identified according to their characteristics (shape, size, etc.), and the level of detail in the segmentation process.

Basically, the type of application defined in the present paper consists of determining, based on space imagery, the area and boundaries of cultivated agricultural plots, according to the spectral response of each crop. An image of the ETM+/ Landsat-7 sensor, WRS 220/74, of August, 14, 2001, was registered using as reference GPS data collected in the field. The registration was performed by means of mathematical determination of the relation between the GPS coordinates points collected in the field and the image coordinates. 


\section{Study Area}

Nascimento (1997) has reported that the processing time for a segmentation using the region-growing algorithm is directly proportional to the size of the image and to the number of bands selected. Hence, initially only the image of the area of interest was extracted, by means of a binary mask equivalent to the total contour of the study area, which corresponds to the territory of three adjacent municipalities north of São Paulo state: Ipuã, Guará, and São Joaquim da Barra $\left(20^{\circ} 17^{\prime}\right.$ to $20^{\circ} 39^{\prime} \mathrm{S}$ and $47^{\circ} 37^{\prime}$ to $48^{\circ} 14^{\prime} \mathrm{W}$ ). This procedure allowed a remarkable reduction in the computer processing time expended in generating each segmentation by the algorithm. Furthermore, it reduced the actual volume of files to be stored, once only the area of interest (approximately 124,000 ha) was used, and not the area of the entire image $(3,240,000 \mathrm{ha})$.

\section{Reference Image}

The methodology for evaluating the segmentation using the empirical method proposed in this paper requires a reference image as a benchmark for quantification of the discrepancy between "reality" and the various segmentations examined in this paper. The reference image attempts to adequately identify and represent the agricultural plots (within the limitations of spatial and spectral resolution), the topographic conditions of the terrain, and the phenologic stage of the crops. It is considered that an "automatically" segmented image will present "discrepancies" with the reference image. Such discrepancies occur when there is non-conformity in regard to the number, size, shape, position, or configuration of the segments produced.

In the present work two reference images were obtained, in two distinct ways: (1) using plot contours obtained in the field (field reference) using a GPS; and (2) using plot contours obtained directly from the computer screen (screen reference); by viewing the ETM+ image, the lines of the plot boundaries were drawn via interpretation directly on the computer screen.

A small reference area, representative of the diversity of the entire study area was defined, instead of using the complete study area. A significant reduction of processing time was thus obtained, and field work was optimized.

\section{Segmentation by the Region-Growing Approach}

Burrough and McDonnell (1998) have claimed that the operator may introduce errors during the process of digitalization of a map's boundaries when using a geographic information system (GIS). In the case of agriculture, the digitalization of agricultural boundaries becomes an expensive task when working with areas that are the size of a county, state, or even larger.

In order to make more automatic the process of extracting the boundaries of agricultural plots and to more efficiently utilize computer resources, the option of image segmentation using the region-growing approach is possible, as implemented in the SPRING software (Câmara et al., 1996). This algorithm generates, by the end of the segmentation process, uniform regions identified by closed segments, and is suitable for regions in which the use of the soil is typically for agriculture, and where each plot has a uniform interior, with the same crops, and whose boundaries are well defined in the field. Furthermore, the region-growing approach allows the user to intervene in 
the segmentation process, by defining thresholds that are most appropriate for the specific application planned.

\section{Optimization of the Combinations Space}

According to Moura (2000), segmentation by the region-growing approach splits the image into spectrally homogeneous regions. The operation of this algorithm requires the definition of the spectral bands and of the similarity and area thresholds. These parameters are responsible for the quality of the segmentation.

Bands $1-5$ plus band 7 of the ETM + sensor were used in all the segmentations performed in the present study.

The RGA segmentation algorithm implemented in the SPRING 3.5 software used here allows a threshold variation from 1 to 50 , in unitary intervals, for the similarity threshold as well as for the area threshold. Thus, there are a large number of possible combinations for the similarity and area thresholds $(50 \times 50=2,500$ possibilities). Therefore, an optimization process to identify which threshold pair will better serve the defined objective becomes necessary. The methodology of optimization, which is the main objective of the present research, is performed according to the following sequence.

1. In a first stage (Fig. 2), the 2,500 possible combinations of similarity and area thresholds are represented by a space comprising 25 cells ( $\mathrm{C} 01, \mathrm{C} 02, \ldots, \mathrm{C} 25)$ with 100 threshold combinations each. Each cell will then be represented by a unique central element, with its corresponding threshold pair, as illustrated in Figure 2. In this way, 25 segmentations are then performed, one for each central pair of thresholds, so as to find the pair with the best result (according to IAVAS - to be defined below).

2. In a second stage, the cell that presented the best result according to IAVAS is partitioned into four equal parts. The scheme illustrated in Figure 2 shows that the cell with the best result was $\mathrm{C} 01$, and in the second stage it was therefore subdivided in four quadrants (Q1, Q2, Q3, and Q4). In this way, the pairs of threshold for the center of each quadrant of cell $\mathrm{C} 01$ are identified, and subsequently only four segmentations are performed, corresponding to the central threshold pair of each quadrant. Among these four segmentations, the one showing the best result according to IAVAS is selected, and in Figure 2, it was quadrant Q1's central pair.

3. The third stage in the process of searching for the best pair of thresholds consists of performing the other 25 segmentations, now considering the threshold pairs of the best quadrant, which, in the example of Figure 2, was quadrant Q1.

Up to this point a total of 53 segmentations were performed ( 25 segmentations in the first stage +4 segmentations in the second stage +24 segmentations in the third stage); therefore, 53 segmentations for the IAVAS index are now available. The next step is to identify, among the 53 segmentations performed, the one with the lowest IAVAS value, which represents the best segmentation result. Note that instead of performing all 2,500 possible segmentations or randomly searching for the best segmentation, as it is common practice, through a "trial and error" process, with only 53 segmentations it was possible to find the best threshold combination for the userdefined application. 

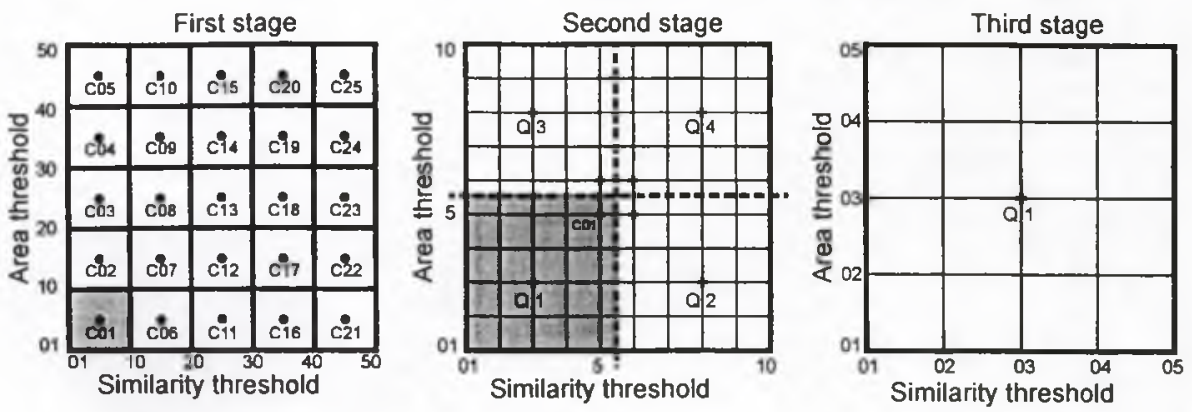

Fig. 2. Stages of the best thresholds determination process for digital image segmentation.

\section{Quantitative Analysis}

According to Nascimento (1997), objective and quantitative evaluation becomes especially necessary for the RGA, considering that there is no ideal value for the similarity and area thresholds, and that they vary according to the imagery used and the segmentation objective. Nascimento also emphasizes that the acceptance or refusal of the thresholds is normally subjective and visually decided by the user, by overlaying the segmented image onto the original image studied.

Consequently, in the present study the RGA algorithm was quantitatively evaluated by measuring the discrepancy between a reference image and the segmented images, considering 53 pairs of thresholds defined according to the optimization method defined previously and depicted in Figure 2. The IAVAS consists of five parameters: Number of Polygons, Total Length of Lines, Variance of Polygon Areas, Nearest Polygon Center, and Coincidence Band; this index is used in the identification of the best segmentation in each stage of the aforementioned optimization method. These five parameters are described in detail below.

1. Number of Polygons (NP). Considering the type of application desired, the number of relevant objects (plots) to be identified by the segmentation algorithm will depend on the selected thresholds (similarity and area). The manipulation of these thresholds can bring the segmentation result to adequate levels (according to the user's objectives) with respect to the number of identified objects (plots). Therefore, the NP parameter seeks to measure the discrepancy between the segmented image and the reference image relative to the quantity of extracted objects detected by the segmentation algorithm. The difference between the value obtained in the segmented image and the reference image may reveal a segmentation that is excessive or, on the other hand, insufficient, depending on the type of application defined (Fig. 3A).

2. Total Length of Lines (L). This parameter allows comparison of different segmentation results that, if analyzed considering only the NP, or even other parameters, could be considered identical. Figure $3 \mathrm{~B}$ illustrates a situation in which the images (segmented and reference) have the same NP, but the L parameter is higher in the segmented image (LS) than in the reference image (LR).

3. Variance of the Polygon Areas (VA). This parameter allows the identification of different segmentation results that, when analyzed considering only the parameters $\mathrm{NP}$ and $\mathrm{L}$, could be considered identical. Figure $3 \mathrm{C}$ is an example of a situation in 


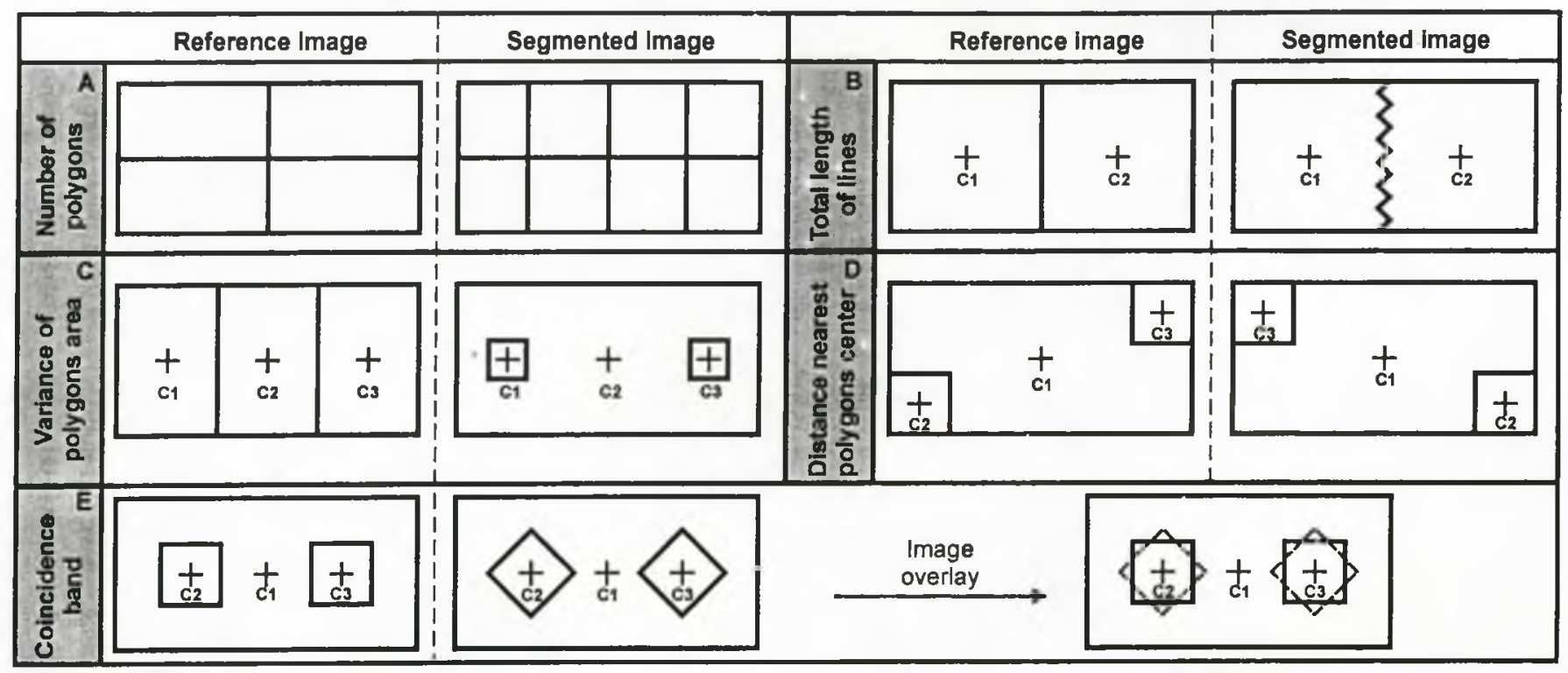

Fig. 3. Discrepancy parameters that constitute the IAVAS index. 
which the reference and the segmented images will be considered different when analyzed according to the VA parameter.

4. Distance to the Nearest Polygon Center (C). The discrepancy parameters described so far do not guarantee that the segments obtained in the automatically segmented image and in the reference image will be spatially coincident. Therefore, a parameter to determine the coherence of the spatial location of segments in both images is necessary. The parameter distance to the nearest polygon center attempts to estimate this coherence by locating spatially the centers of the polygons. Considering the Euclidean distance separating the position of the polygons' centers in the reference image from their position in the segmented image, it is possible to analyze the results of the different segmentation processes that would be considered identical if only the NP, L, and VA parameters were considered. An example of this situation is represented in Figure 3D, where the reference and segmented images are considered different when parameter $\mathrm{C}$ is analyzed.

5. Coincidence Band (FC): The four discrepancy parameters referred to above have thus far assured a coherence among the polygons in relation to quantity, profile, size, and position of the segments within the images. However, in situations in which the segments are rotated in relation to their center, the parameters above are not capable of identifying the discrepancy in the location of their limits. Thus, in order to better evaluate the similarity between the position of the segment edges in the segmented image and in the reference image, the Coincidence Band of the segment edges of the images was established as a fifth parameter. Determination of this parameter involves quantifying the pixels cut by the segment boundaries in the segmented image that coincide with the pixels cut by the segment boundaries in the reference image. Such a procedure is performed by converting the vectorial data of the limits into matrix form, which allows the overlaying and comparison of the images. Figure $3 \mathrm{E}$ shows a situation in which the reference and the segmented images are considered different when analyzed according to the parameter FC.

\section{Index for the Evaluation of Segmentation (IAVAS)}

The five discrepancy parameters that composing the IAVAS index have distinct scales and units. Consequently, for the evaluation index to reveal uniformly the parameters' influence, it is necessary to normalize the data for each parameter before merging them into a single unique index.

The normalization process of a set of values is performed to homogenize variables of different scales and different behaviors. In general, the set of values is transformed in order to adjust them to an approximately normal (or Gaussian) distribution with an average equal to zero and variance equal to one. This is obtained by subtracting from each observed value $\left(\mathrm{X}_{\mathrm{i}}\right)$ the value of the average, then dividing the result by the standard deviation (S). The normalization does not necessarily produce a distribution with average of zero, so in the present study the transformation chosen is one that causes the transformed average to assume any value providing that it guarantees that the minimum value found in the original value set would assume a value of zero in the set of the transformed data. It was obtained by subtracting from each observed value the smallest value in the whole set $\left(\mathrm{X}_{\text {minimum }}\right)$ and then dividing by the standard deviation, as shown in Equation 1. 
Equation 1 describes the IAVAS index and its constituent discrepancy parameters. The values obtained by Equation 1 are greater than or equal to zero, the null value representing an optimal segmentation, or of maximum quality; non-null values mean loss of quality, as the larger the IAVAS value, the greater the loss.

$$
\begin{gathered}
\text { IAVAS }=\left\{\left[\frac{\left(\left|\mathrm{NP}_{\mathrm{R}}-\mathrm{NP}_{\mathrm{S}}\right|\right)-\mathrm{MVDA}_{\mathrm{NP}}}{\mathrm{DPDA}_{\mathrm{NP}}}\right]+\left[\frac{\left(\left|\mathrm{L}_{\mathrm{R}}-\mathrm{L}_{\mathrm{S}}\right|\right)-\mathrm{MVDA}_{\mathrm{L}}}{\mathrm{DPDA}_{L}}\right]+\right. \\
{\left[\frac{\left(\left|\mathrm{VA}_{\mathrm{R}}-\mathrm{VA}_{\mathrm{S}}\right|\right)-\mathrm{MVDA}_{\mathrm{VA}}}{\mathrm{DPDA}_{\mathrm{VA}}}\right]+\left[\frac{\left(\mathrm{C}_{\mathrm{R}-\mathrm{S}}\right)-\mathrm{MVDA}_{\mathrm{C}}}{\mathrm{DPDA}_{\mathrm{C}}}\right]+} \\
\left.\left[\frac{\left(\left|\mathrm{NQ}_{\mathrm{R}}-\mathrm{NQ}_{\mathrm{S} \cong(\mathrm{R}+\mathrm{FC})}\right|\right)-\mathrm{MVDA}_{\mathrm{FC}}}{\mathrm{DPDA}}\right]\right\},
\end{gathered}
$$

where $\mathrm{NP}_{\mathrm{R}}$ and $\mathrm{NP}_{\mathrm{S}}$ are the number of polygons of the reference and segmented images, respectively; $L_{R}$ and $L_{S}$ are the total length of lines present in the reference and segmented images; $\mathrm{VA}_{\mathrm{R}}$ and $\mathrm{VA}_{\mathrm{S}}$ are variance in the areas of the polygons of the reference and segmented images; $\mathrm{C}_{\mathrm{R}-\mathrm{S}}$ is the average of the smaller distances separating the centers of the reference image polygons and the centers of the segmented image polygons; $N Q_{R}$ is the number of elements in the reference image; $N Q_{S \simeq(R+F T)}$ is number of elements in the segmented image coinciding with the elements of the reference image plus the Coincidence Band (FC); MVDA is the lowest value for the absolute differences in the analyzed parameter; and DPDA is the standard deviation of the absolute differences for the analyzed parameter.

\section{RESULTS AND DISCUSSION}

A unique pair of similarity and area thresholds was obtained from the tested area. This pair of thresholds offered the best result in the segmentation process, and are the values to be applied to the whole scene segmentation.

\section{Field Reference versus Screen Reference}

A fundamental requirement for the application of IAVAS is the availability of two images that allow us to measure the discrepancies (that is, the differences between the actual boundaries in the field versus the boundaries obtained through automatic segmentation). The first image is the spatial division performed by the segmentation algorithm product, and the second image is the spatial division performed by the operator, which is considered the reference data. Both images are fundamental for a good evaluation of the segmentation.

The present work evaluated, through the IAVAS index, the automated segmentation of the test area by comparing it with two sources of reference data- the field reference and the screen reference. The best results were obtained with a similarity threshold equal to 16 for both reference images, and with an area threshold equal to 24 for the field reference and equal to 44 or 45 (there was a tie according to the IAVAS) using the screen reference image. 
Table 1. Best IAVAS Results according to Number of Analyzed Segmentations

\begin{tabular}{ccc}
\hline \hline Segmentation/combination & Field reference & Screen reference \\
\hline 53 segmentations $^{\mathrm{a}}$ & ImgSeg1240 & ImgSeg1644 \\
& & ImgSeg1645 \\
184 segmentations $^{\mathrm{b}}$ & ImgSeg1240 & ImgSeg1634 \\
\hline
\end{tabular}

a Defined by the optimization stages of combination space $\geq$ IAVAS.

bRandom combinations.

It was possible to verify that the use of the screen reference image was most useful, once the extraction of the reference limits is directly related to the operator's interpretation of the image shown in his/her computer screen. Furthermore, the boundaries are identified according to the spectral response of the target object, which is fundamental for the decision criteria of the region-growing algorithm. So, physical boundaries existing in the field but that are imperceptible to the sensor (e.g. dirt roads connecting plots in which the soil has been prepared for sowing) are not introduced into the reference image, which directly contributes to the quality of the evaluation.

The advantages of the screen reference are superior and more adequate for the process of evaluation of the automatic segmentation of space imagery. Among these advantages are: (1) broad view of the study area; (2) low cost, as the use of precision devices (e.g., GPS) or a trip to the field is not necessary; and (3) the possibiiity of using image processing techniques (contrast- and edge-enhancing filters, false-color composite techniques) to enhance the spectral limits of the target objects.

\section{Efficiency Analysis of the Optimization Process}

The procedure of optimizing the number of threshold combinations allowed a significant reduction in the number of tests necessary to determine threshold pairs (from 2,500 to 53 segmentation tests), thus affording a significant reduction in computing time. Nevertheless, it may be acknowledged that the most proper way to verify this efficiency would be to evaluate all 2,500 possible segmentations for the test area and to identify the best absolute result through the IAVAS index: i.e., it should be verified that the best result obtained from the 53 segmentations was the same as that identified by evaluating all 2,500 segmentations.

Given the great computing effort and time necessary to establish the absolute result among the 2,500 possible combinations, 184 random combinations were performed, to analyze the behavior of the IAVAS index.

Table 1 presents the best segmentations defined by the IAVAS index according to the number of combinations analyzed. There is some divergence in the best results, due to the low number of combinations analyzed. The expression "ImgSeg1240" means: ImgSeg (segmented image), 12 (value of the similarity threshold), and 40 (value of the area threshold).

It is possible to verify that the indication of the best pair of thresholds diverged when the proposed method of subdivision of the combinations space was used versus when the random sample of this space was used, at least in the case of the screen 


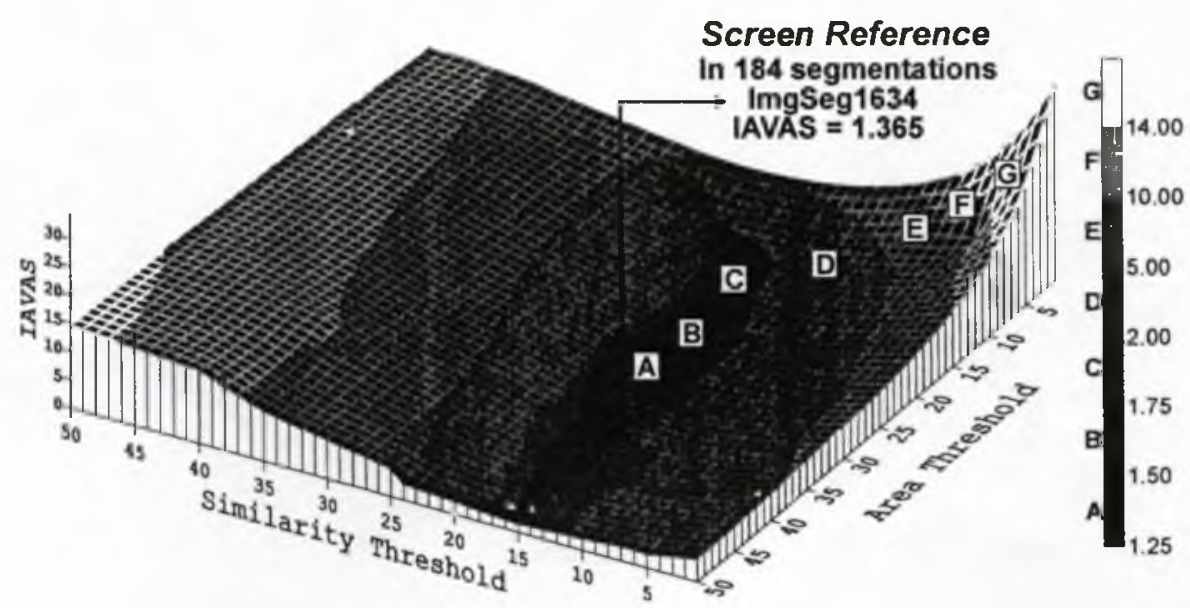

Fig. 4. Behavior of the IAVAS index (184 combinations of thresholds) based on the screen reference image for the test area.

reference (interpretation of the computer screen). Once it was determined that the result obtained by the optimization of the combinations space diverged from the result obtained with a larger number of (random) combinations, the next step was to evaluate whether the differences actually found could alter significantly the quality of the segmentation calculated according to the IAVAS index.

With this intention, the variation of the IAVAS index among the 184 pairs of thresholds was analyzed, using the screen reference image. The result of this analysis is shown in Figure 4. According to the analysis of the divergences between the image segmentations defined in Table 1 and the screen reference, and through the analysis of the behavior of the IAVAS index according to the variation of the similarity and area threshold shown in Figure 4, it is possible to verify that the procedure for the optimization of the combinations space provided excellent results in the process of segmentation evaluation. Such efficiency is proved by the following indicators.

1. The process of optimization of the combinations space used only 53 pairs of thresholds (only $2.12 \%$ ) among the 2,500 possible combinations of the regiongrowing algorithm used.

2. ImgSeg1644 and ImgSeg 1645, obtained with the application of IAVAS, are located in the region of the lowest IAVAS indexes as shown in Figure 4, and consequently, in the region where the combinations offered the best segmentations according to the screen reference image.

3. The lowest IAVAS index among the 184 segmentations based on the screen reference was 1.365, presented for ImgSeg1634. ImgSeg1644 and ImgSeg1645, defined by the optimization procedures, had an IAVAS value of 1.449 , a difference of only 0.084 from the best index. Small alterations separating the similarity and area thresholds result in differences of little significance on the IAVAS index. Consequently, the difference of only 0.084 proves that the optimizing the combinations space is an efficient process for identifying the combinations of thresholds. 


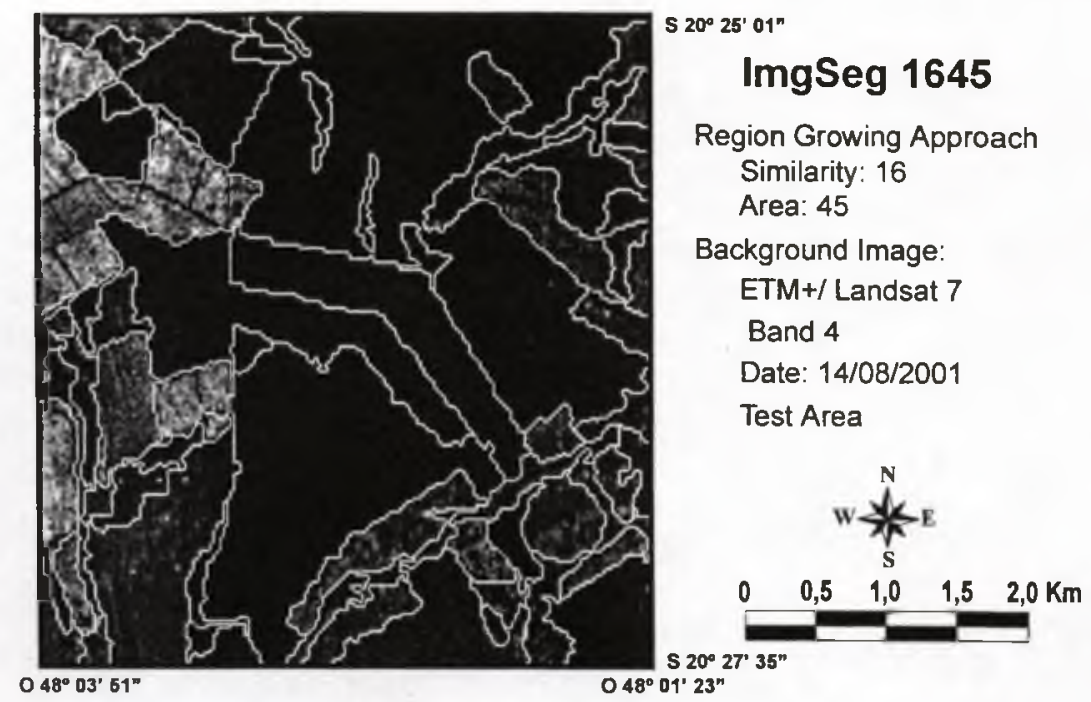

Fig. 5. Segmented image using thresholds $16 / 45$ superimposed on a section of an ETM+ space image of the test area.

\section{Segmentation of the Study Area}

The above subsection of the paper confirms the efficiency of the combinations space for the optimization process, as evaluated by the IAVAS index. The threshold values determined according to the respective reference images hence are in accordance with the type of application established for the segmentation.

Based on the advantages of the reference image produced by the operator's interpretation (screen reference) and described above, the values chosen for the similarity and area thresholds were, respectively, 16 and 45, representing adequate values for segmenting the study area. These thresholds were obtained after an evaluation of the screen reference image using the IAVAS index. Figure 5 shows the behavior of IAVAS applied to 184 pairs of thresholds (similarity and area), based on the screen reference for the test area.

\section{CONCLUSIONS}

The use of the region-growing segmentation approach using the ETM $+/$ Landsat image proved to be efficient for the delimitation of agricultural objects present on the scene, according to the similarity and area thresholds indicated by the IAVAS evaluation process. Lower area threshold values resulted in excessive partitioning, producing a confused visual picture of the regions. On the other hand, excessively high similarity threshold values clustered spectrally distinct regions in the same segment, causing partial or total loss of some regions of interest.

The quantitative and objective method developed for the evaluation of the segmentation, represented by the Index for the Evaluation of Segmentation (IAVAS), proved to be an efficient method for evaluating the results produced by the segmentation algorithm, distinctively identifying the threshold pairs that produced values 
coherent with the reference image. The parameters of discrepancy compounding the IAVAS index (number of polygons, total length of lines, variance of polygon areas, nearest polygon center, and coincidence band) proved effective in identification of the number, quantity, size, and position of the target object in the segmented image according to the type of application established for this work.

The objective of the segmentation, established according to end user need, in this study was the delimitation of agricultural plots on an ETM $+/$ Landsat space image. The need to perform a field survey on a date close to the acquisition of the image to be segmented, associated to the complexity of the data survey (cost of equipment, weather and topographic conditions, difficulty of access to the target area, and great distances to be traveled) rendered impractical the use of the field reference in the process of evaluating the segmentation, despite the tendency of a reference image developed from a field survey to provide information that closely approximates the actual image. Furthermore, when using the field reference, the possible introduction in the image of boundaries on the ground that do not constitute spectral boundaries on the image causes a significant loss to the quality of the evaluation, since the IAVAS index would be evaluating the segmentation image based on a reference image that is inconsistent.

On the other hand, the screen reference proved to be practical, swift, and accurate, and produced results that were coherent with field reality, without the inconveniences of the field reference. This greater efficiency is attributed to the operator's visual interpretation, once he/she uses the scene spectral response as a criteria for decision during manual segmentation. The segmentation algorithm also uses this response to extract the boundaries of an image.

The optimization process in the combinations space proved its efficiency by identifying, in association with the IAVAS index, pairs of thresholds with satisfactory results according to an applied reference image, with a set of only 53 segmentations, which represents $2.12 \%$ of the 2,500 possible combinations of similarity and area thresholds of the region-growing segmentation algorithm used. As in the current study, in which the objective of the segmentation applied to an agricultural area was the delimitation of plots for the purpose of examining the cultivated surface, the IAVAS index may be applied to a wide variety of situations. This flexibility is directly related to the screen reference, since the visual interpretation by the user allows the adjustment of the reference image for a number of other situations, according to the type of application established for the segmentation.

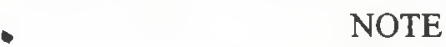

'More information about the region-growing approach segmentation algorithm may be obtained in the work of Bins et. al. (1996), Nascimento (1997), and INPE (2001).

\section{REFERENCES}

Bins, L. S., L. M. G. Fonseca, G. J. Erthal, and F. M. Li. "Satellite imagery segmentation: A region-growing approach," in: Simpósio Brasileiro de Sensoriamento Remoto, 8., Salvador, abr. 1996. Anais. São José dos Campos, Brazil: INPE, 1996. Sessão de Comunicações Técnico-Científicas. Repositório da URLib 
[sid.inpe.br/deise/1999/02.05.09.30 and http://iris.sid.inpe.br:1908/rep/sid.inpe. br/deise/1999/02.05.09.30], accessed June 10, 2002.

Borsotti, M., P. Campadelli, and R. Schettini. "Quantitative evaluation of color image segmentation results," Pattern Recognition Letters, Vol. 19, No. 8, 1998, pp. 741-747.

Burrough, P. A. and R. A. McDonnell. Principles of Geographical Information Systems. New York, NY: Oxford University Press, 1998, 333 p.

Câmara, G, R. C. M. Souza, U. M. Freitas, and J. C. P. Garrido. "SPRING: Integrating remote sensing and GIS with object-oriented data modelling," Computers and Graphics, Vol. 15, No. 6, 1996, pp. 13-22.

Gonzalez, R. C., and P. Wintz. Digital Image Processing. Reading, MA: Addison Wesley, 1987, $431 \mathrm{p}$.

Haralick, R. M. and L G. Shapiro. Image Segmentation Techniques. Ann Arbor, MI: Machine Vision International, 1984, 245 p.

INPE (Instituto Nacional de Pesquisas Espaciais). Imagem de satélite Landsat ETM+ - ORB 220 P 074 [CD ROM]. Cachoeira Paulista, Brazil: Divisão de Geração de Imagens (DGI), 2001.

INPE (Instituto Nacional de Pesquisas Espaciais). Manual do SPRING: Registro [http://www.dpi.inpe.br/spring/usuario/registro.htm], January 2002.

Moura, V. Imagens multitemporais TM/LandSat-5 da época de pré-plantio na estimativa de áreas destinadas a culturas de verão. Unpubl. master's dissertation, Instituto Nacional de Pesquisas Espaciais, São José dos Campos, INPE-7527- TDI/ 734, 2000, $122 \mathrm{p}$.

Nascimento, P. S. R. Avaliação de técnicas de segmentação e classificação por regiões em imagens Landsat-TM visando o mapeamento de unidades de paisagem na Amazônia. Unpubl. master's dissertation, Instituto Nacional de Pesquisas Espaciais, São José dos Campos, INPE-6391-TDI/607, 1997, 120 pp.

Schoenmakers, R. P. H. M., G. G. Wilkinson, and T. E. Schouten. "Segmentation of remotely-sensed images: A re-definition for operational applications," in: 1991 International Geoscience and Remote Sensing Symposium, IGARSS'91, Espoo, Finland, June 3-6, 1991, Vol. 2, 1991, p. 1087-1090.

$\mathrm{Xu}, \mathrm{Y}$. V. Olman, and C. Uberbacher. "A segmentation algorithm for noisy images: Design and evaluation," Pattern Recognition Letters, Vol. 19, No. 13, 1998, p. 1213-1224.

Woodcock, C. E., and V. J. Harward. "Nested-hierarchical scene models and image segmentation," International Journal of Remote Sensing, Vol. 13, No. 16, 1992, p. 3167-3187.

Woodcock, C. E., J. B. Collins, S. Gopal, V. D. Jakabhazy, X. Li, S. Macomber, S. Ryherd, V. J. Harward, J. Levitan, Y. Wu, and R. Warbington. "Mapping forest vegetation using Landsat TM imagery and a canopy reflectance model," Remote Sensing of Environment, Vol. 50, No. 3, 1994, p. 240-254.

Zhang, Y. J. "A survey on evaluation methods for image segmentation," Pattern Recognition Letters, Vol. 29, No. 8, 1996, pp. 1335-1346.

Zhang, Y. J. "Evaluation and comparison of different segmentation algorithms," Pattern Recognition Letters, Vol. 18, No. 10, 1997, p. 963-974.

Zucker, S. W. "Region growing: childhood and adolescence," Computer Graphics and Image Processing, Vol. 5, No. 3, 1976, p. 382-399. 